Revista Electrónica de Investigación en Ciencias Económicas

Abriendo Camino al Conocimiento

Facultad de Ciencias Económicas, UNAN-Managua

\title{
USO Y EXPLOTACION DE LAS TIERRAS EN NICARAGUA
}

Ariana Karelya Castro Sobalvarro

Estudiante 5to año carrera economía

UNAN-Managua

arianacstro22@gmail.com

Grethel Carolina Requene Acosta

Estudiante 5to año carrera economía

UNAN-Managua

ethelacoste1494@gmail.com

Fecha recepción: mayo 25 del 2015

Fecha aceptación: junio 6 del 2015

Palabras claves: Actividad Agropecuaria,

Explotación Agrícola, Uso de la Tierra, cultivos, seguridad alimentaria

Key words: Agricultural Activity, Holding, Land Use, crops, food security

ISSN: $2308-782 X$

\section{REICE}

Revista Electrónica de Investigación en Ciencias Económicas http://revistacienciaseconomicas.unan.edu.ni

revistacienciaseconomicas@gmail.com revistarucfa@unan.edu.ni

\section{Resumen}

El objetivo de este ensayo es dar a conocer el uso y explotación de la tierra en el sector agrícola nicaragüense. El método utilizado es de carácter descriptivo. Los datos provienen del IV Censo Agropecuario del INIDE, y para mayor comprensión se hará un acercamiento de variables utilizadas en los resultados de la encuesta. Los resultados obtenidos del análisis indican como ha sido la explotación y uso del suelo y se pueden mencionar: La crianza de aves, ganado; explotaciones en la actividad agrícola, manzanas sembradas con cultivos permanentes y semi-permanentes, siembra de granos básicos, etc. Se concluye que la encuesta brinda información del sector agropecuario e investiga variables nuevas de interés para el país como: Actividad principal de la Explotación Agropecuaria, tenencia de las parcelas, actividad acuícola en las Explotaciones Agropecuarias, característica de los miembros del hogar del productor(a) y seguridad alimentaria.

\section{Abstract}

The aim of this paper is to present the use and exploitation of land in the Nicaraguan agricultural sector. The method used is descriptive. The data come from the Census of Agriculture INIDE IV, and to greater understanding will be a rapprochement of variables used in the survey results. The results of the analysis indicate as has been the exploitation and use of land and include: The poultry, cattle; agriculture farms, apples planted with permanent and semi-permanent crops, basic grains, etc. It is concluded that the survey provides information on the agricultural sector and investigates new variables of interest for the country such as: Main activity of the farm, tenure plots in aquaculture farms, characteristic of household members producer (a) and food security.
REICE 191 
Revista Electrónica de Investigación en Ciencias Económicas

Facultad de Ciencias Económicas, UNAN-Managua

Uso y Explotación de las tierras en Nicaragua

Introducción

En Nicaragua las categorías de uso de la tierra se clasifican en agrícola, pecuarias, forestales y de protección de los recursos naturales. Cabe señalar que desde tiempos antiguos la población nicaragüense ha hecho uso de los recursos que dispone para el sustento económico y social.

REICE 192

Sin embargo, con el paso del tiempo el uso inapropiado del suelo puede traer consigo consecuencias irremediables, el deterioro podría ser permanente, y con ello se afectaría la producción de materia prima, la población y la economía nacional, por lo tanto se deberán tomar medidas que permitan el buen uso de este.

En la Bibliografía consultada se encontró información relevante para el desarrollo de este tema. "Análisis de la utilización del recurso suelo en Nicaragua", es un escrito de Vivas (2010), quien explica brevemente lo que es la valoración del suelo, la disponibilidad del suelo por habitante haciendo comparación con los países de Centro América, y ejemplifica en términos económicos como la curva de Lorenz y el coeficiente de Gini para hacer su valoración.

Se consultó un documento del MAGFOR (2010), "Uso potencial de la tierra comprendido en Mapas", donde se muestra como ha sido el uso potencial por los distintos sectores, donde se incluyen desde centros poblados hasta áreas protegidas.

Estado actual de la Información sobre Recursos Forestales y cambio en el uso de la tierra Roldan (2002), es parte de un documento titulado "Estado de la información forestal en Nicaragua", con el apoyo de la Unión Europea y La Organización de las Naciones Unidas para la Agricultura y la alimentación.

Se indago sobre leyes que fuesen acorde al tema como tal el "Decreto no. 782 Ley de Reforma Agraria"; dando a conocer que es una necesidad superar las formas de propiedad y explotación de la tierra-rentista extensivas o ineficientes que constituyen un obstáculo al desarrollo y al progreso, así como eliminar la explotación inicua del trabajo campesino bajo las modalidades de mediería, aparcería, colonato y formas similares en su artículo V.

Ley no. 387 "Ley especial sobre explotación y exploración de minas". 


\section{Revista Electrónica de Investigación en Ciencias Económicas \\ Facultad de Ciencias Económicas, UNAN-Managua \\ Uso y Explotación de las tierras en Nicaragua}

Cabe agregar que en la explotación y uso de la tierra también se destacan: Las áreas protegidas, las minas, aprovechamiento de bosques, de árboles, forestación y deforestación, frontera agrícola, extracción ilegal, etc. Por lo tanto también: "Verificación del aprovechamiento forestal en Nicaragua" es otro documento consultado. Donde se detalla causas y consecuencias de los temas antes mencionados.

El propósito es presentar un breve informe del uso adecuado del potencial productivo de la tierra, ya que este puede desarrollar una mejor actividad agropecuaria y forestal sin menoscabo de los recursos naturales, con el fin de fortalecer la producción agrícola.

Con el apoyo del IV Censo Agropecuario realizado por el INIDE y el MAGFOR se analiza la importancia de aprovechamiento de la tierra y el uso adecuado de la misma con el fin de garantizar bienestar a la población.

Material y métodos

La información del IV Censo Nacional Agropecuario fue recolectada del 15 de mayo al 16 de junio de 2011 por el INIDE, en estrecha coordinación con el MAGFOR, conforme Decreto Presidencial No.66-2010.

El Censo brinda información del sector agropecuario, tales como: Número de explotaciones agropecuarias, productores y productoras individuales, tamaño de las explotaciones agropecuarias, la forma de trabajarlas, tenencia y uso del suelo, superficie sembrada, prácticas agrícolas y pecuarias, acceso al crédito, asistencia técnica y capacitación agropecuaria, existencia de ganado mayor y menor, colmenas y mano de obra en el sector agropecuario.

Toda la información brindada en este informe está referida a entrevistas completas. Es decir, que durante el levantamiento de la información se realizaron como muestra 262,974 entrevistas fundamentadas en la Explotación Agropecuaria, siendo entrevistas completas el $97.77 \%$.

El objetivo de este ensayo se resume en presentar un breve informe sobre el uso y explotación de la tierra en Nicaragua, en base al VI Censo Agropecuario realizado por el INIDE. La selección de la muestra se hizo con la técnica de obtención de datos, por medio de la lectura de documentos digitales (PDF, Word, Excel) páginas web y escritos. El tipo de investigación es de orden documental debido a que se realizó en base a publicaciones del INIDE y el MAGFOR. 
Revista Electrónica de Investigación en Ciencias Económicas

Facultad de Ciencias Económicas, UNAN-Managua

Uso y Explotación de las tierras en Nicaragua

A través de los censos estructurales, se obtiene información básica que no cambia constantemente en el tiempo y una unidad estadística de investigación que explica la explotación agropecuaria y posteriormente se hacen periodos de referencia de dicha explotación. A partir de la información proporcionada se extrae información necesaria para la elaboración de análisis en las estadísticas obtenidas.

Conceptos y definiciones básicas

Explotación Agropecuaria (EA): Es todo terreno que se utiliza total o parcialmente para la producción agropecuaria, agricultura, ganadería o la combinación de ambas, que se explota como unidad económica dentro de un municipio; sin consideración del tamaño, régimen de tenencia, ni condición jurídica.

No se considera Explotación Agropecuaria: Los terrenos dados en concesión para la extracción de productos naturales, ya sean vegetales o animales, por ejemplo las explotaciones exclusivamente forestales, cría de especies acuáticas (acuicultura) en forma exclusiva, gallos de pelea, perros y caballos de raza para etiqueta.

Uso potencial del suelo la utilización más apropiada de este recurso natural dentro de cada patrón edafoclimático (condiciones de clima y suelo). Para ello se requiere la aplicación de sistemas adecuados de manejo para que la tierra pueda ser sometida a una explotación sostenida con el mínimo deterioro.

Parcela: Son los terrenos de la Explotación Agropecuaria dentro de cualquier municipio, manejados bajo una sola forma de tenencia, separados por: Ríos o quebradas con agua permanente, Carreteras o caminos públicos, Terrenos productivos o improductivos que no forman parte de la Explotación Agropecuaria.

Productor(a) Agropecuario: Persona Natural o Jurídica,). Toma decisiones respecto a los cultivos, crianza de animales, inversiones, asume riesgos económicos y disfruta de los beneficios.

Actividad principal de la explotación agropecuaria: Uso que se le está dando a la Explotación Agropecuaria. La actividad está en función del uso de la tierra y de la infraestructura económica existente en estas.

Exportación tradicional: Aquellos productos tale como café, ajonjolí, maní, algodón, etc., que se cultivan con el propósito de exportarlos fuera del país. 
Revista Electrónica de Investigación en Ciencias Económicas

Facultad de Ciencias Económicas, UNAN-Managua

Uso y Explotación de las tierras en Nicaragua

Exportación no tradicional: Productos como melón, pitahaya, limón Tahití, etc. Estos cuando se cultivan reciben un tratamiento que permite clasificarlos como tales. Generalmente se siembran para exportarlos.

Ganadería de leche: Cuando el propósito del hato es la producción de leche o sus derivados (queso, crema, etc.).

Áreas protegidas: Es un espacio geográfico claramente definido, reconocido, dedicado y gestionado, mediante medios legales $u$ otros tipos de medios eficaces para conseguir la conservación a largo plazo de la naturaleza y de sus servicios ecosistémicos y sus valores culturales asociados.

Frontera agrícola: Es el límite que divide la tierra dedicada a la agricultura y la tierra que aún se mantiene como área natural intacta. Debido a la presión de las poblaciones humanas, esta frontera avanza cada vez más hacia las áreas naturales.

Ley de reforma agraria: Una reforma agraria es un conjunto de medidas políticas, económicas, sociales y legislativas impulsadas con el fin de modificar la estructura de la propiedad y producción de la tierra.

\section{Resultado y Discusión}

El suelo en Nicaragua se puede valorar desde una perspectiva productiva, económica, social, organizativa e histórica, por lo tanto su uso es indispensable para la economía y su explotación para el bienestar de la sociedad siempre y cuando no se derroche bruscamente su potencial.

Es el país que tiene la más alta disponibilidad de suelo por habitante en Centroamérica, y en caso de relacionarlo con la superficie total El Salvador es el que tiene la disponibilidad más baja por persona; por tal situación el poseedor de estas es privilegiado (Vivas, 2005).

Tiene el $82.2 \%$ del suelo de uso agropecuario, pero, la estructura de uso del área agropecuaria, se encuentra un uso extensivo. El $39.9 \%$ es de pasto natural y el $23.1 \%$ de suelo en descanso y tacotales. Este es un ángulo de importancia, debido a la creciente necesidad de alimentos y conservación de los recursos naturales. 
Revista Electrónica de Investigación en Ciencias Económicas

Facultad de Ciencias Económicas, UNAN-Managua

Uso y Explotación de las tierras en Nicaragua

Grafica $\mathrm{N}_{0:} 1$

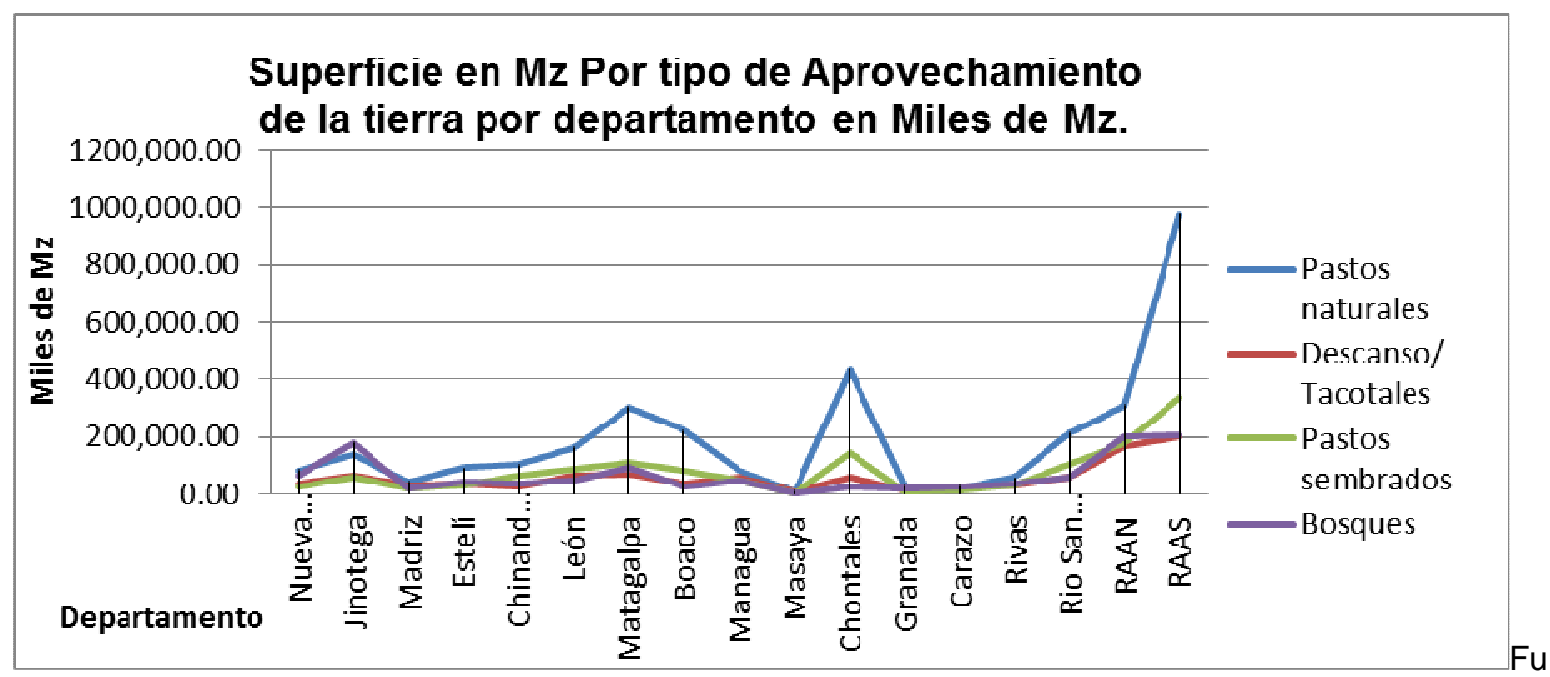

REICE

ente: Elaboración propio en base al IV Cenagro Agropecuario, INIDE.

La mayor cantidad de superficie la ocupan los pastos naturales, con un total de 3 , 287, $755.79 \mathrm{mz}$ destacándose las Regiones del Atlántico con 1, 287, 412.69; en cambio la explotación de los bosques es devastadora por la gran necesidad del uso de la tierra que alcanza las $200,000 \mathrm{mz}$, casi no se conservan bosques ya que el descanso de las tierras y los pastos sembrados, están a un margen mínimo de diferencia con la explotación de los bosques.

De las tierras arables que tiene Nicaragua se espera que se pierdan más hectáreas de bosques, lo que significa que un alto porcentaje del suelo se deforesta cada año. Este es un problema grave que se le debe prestar atención (Vivas, 2005).

En los resultados del Censo Agropecuario 2011; se observa un primer panorama sobre el uso del suelo, en el que se puede ver que el peso porcentual de las explotaciones aumenta sistemáticamente en las distintas propiedades, teniendo implicaciones económicas y sociales. Esta realidad presupone el diseño de políticas que mejoren la eficiencia de uso del suelo.

Por consiguiente, el suelo es uno de los principales recursos naturales que presenta las condiciones físicas, biológicas y químicas para que los cultivos desarrollen sus etapas fisiológicas y la respectiva producción. 
Revista Electrónica de Investigación en Ciencias Económicas

Facultad de Ciencias Económicas, UNAN-Managua

Uso y Explotación de las tierras en Nicaragua

En la agricultura es objeto de trabajo, medio de trabajo y por ende medio de producción. Por eso siempre tiene un rol que jugar, sabiendo que no podemos producir más suelo del que se posee.

El panorama general del país es el punto de partida para realizar un análisis exhaustivo sobre cómo se está aprovechando su potencial, el $83.74 \%$ del suelo es agropecuario. El $38 \%$ es de pasto natural y el $11.59 \%$ de suelo en descanso y tacotales ${ }^{1}$. Este es un ángulo de importancia, debido a la creciente necesidad de alimentos y conservación de los recursos naturales.

Tabla No: 1 Uso del suelo actual en Nicaragua

\begin{tabular}{|l|r|r|}
\hline \multicolumn{1}{|c|}{$\begin{array}{c}\text { Aprovechamiento } \\
\text { De la tierra. }\end{array}$} & $\begin{array}{c}\text { Superficie } \\
\text { utilizada }\end{array}$ & $\begin{array}{c}\text { Porcentaje } \\
\text { \% }\end{array}$ \\
\hline Cultivos anuales y temporales & $1,045,699.88$ & $12.18 \%$ \\
\hline $\begin{array}{l}\text { Cultivos permanentes y semi- } \\
\text { permanentes }\end{array}$ & $510,129.01$ & $5.93 \%$ \\
\hline Tierras en descanso (tacotales) & $995,573.95$ & $11.59 \%$ \\
\hline Pastos naturales & $3,287,755.79$ & $38 \%$ \\
\hline Pastos cultivados & $1,352,953.81$ & $15.75 \%$ \\
\hline Bosques & $1,139,721.42$ & $13.3 \%$ \\
\hline Instalaciones y viales & $99,866.13$ & $1.16 \%$ \\
\hline Pantanos, pedregales, otros & $156,766.91$ & $1.82 \%$ \\
\hline Superficie total & $\mathbf{8 , 5 8 8 , 4 6 6 . 9 0}$ & $\mathbf{1 0 0 \%}$ \\
\hline
\end{tabular}

Fuente: IV censo Agropecuario 2011 INIDE.

El potencial para las actividades agrícolas se presenta mayoritariamente en la Región del Pacífico y los valles intramontanos de la Región Central. En esta última predomina el potencial forestal seguido del potencial ganadero y agrícola. En la Región del Caribe el potencial es eminentemente forestal con actividades agropecuarias bajo sistemas agroforestales y silvopastoriles. ${ }^{2}$

En el siguiente mapa se muestra como está distribuido el uso potencial del suelo en Nicaragua y como este se explota a diario para obtener ingresos económicos nacionales e individuales.

\footnotetext{
${ }^{1}$ IV Cenagro Agropecuario "Superficie en mz de la tierra por tipo de aprovechamiento, según tamaño de las explotaciones agropecuarias" pág. 14.

2. "Uso Potencial de la tierra comprendido de mapas". MAGFOR, INETER. Pág. 25 y 26.
} 
Revista Electrónica de Investigación en Ciencias Económicas

Facultad de Ciencias Económicas, UNAN-Managua

Uso y Explotación de las tierras en Nicaragua

Hacer referencia al mapa es importante para tomar en cuenta cómo, dónde y de qué manera Nicaragua genera producción agrícola y conociendo como se distribuye en el territorio nacional la producción según su delimitación geográfica, clima y ambiente, para cultivos perennes, semi-perennes, anules, temporales etc.

Mapa $\mathrm{N}_{0: 1}$ Uso potencial de la tierra en Nicaragua.

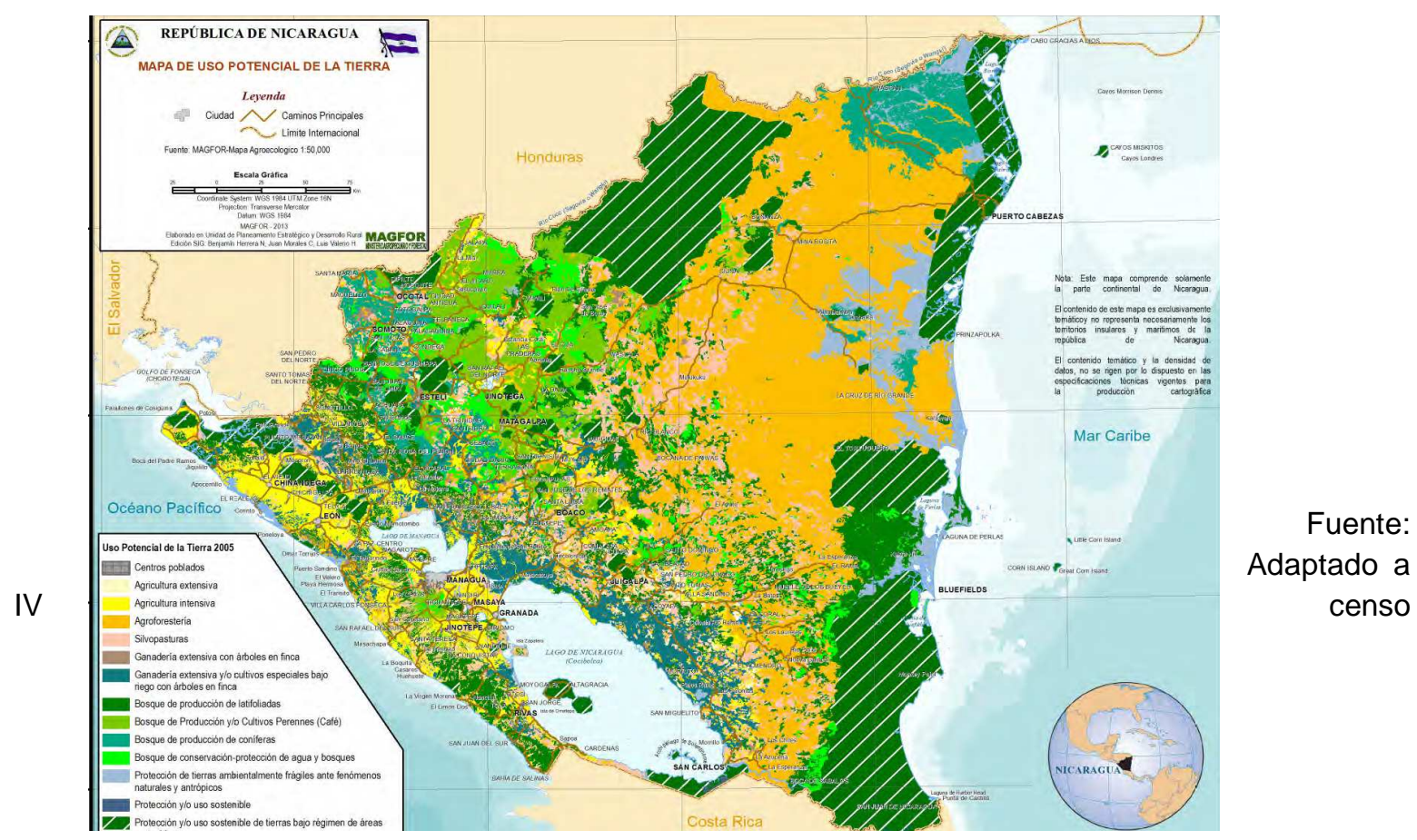

Agropecuario MAGFOR- INETER

Aquí se muestran: Centros poblados, Agricultura extensiva, Agricultura extensiva, Agroforestería, silvopasturas, Ganadería extensiva con árboles en finca, ganadería extensiva y/o cultivos bajo riego con árboles en finca, bosque de producción de latifoliadas, bosque de producción y/o cultivos perennes (café), bosque de producción de coníferas, bosque de conservación- protección de agua y bosques, protección de tierras ambientalmente ante fenómenos naturales y antrópicos, protección y/o uso sostenible y protección o uso sostenible de tierra bajo régimen de áreas protegidas. 
Revista Electrónica de Investigación en Ciencias Económicas

Facultad de Ciencias Económicas, UNAN-Managua

Uso y Explotación de las tierras en Nicaragua

Tabla $\mathrm{N}_{0}: 3$ Aprovechamiento de la tierra según tamaño de la EA.

\begin{tabular}{|l|c|c|c|c|}
\hline $\begin{array}{c}\text { Tamaño de las } \\
\text { Explotaciones } \\
\text { Agropecuarias }\end{array}$ & $\begin{array}{c}\text { Total de la } \\
\text { Explotación } \\
\text { Agropecuaria. }\end{array}$ & $\begin{array}{c}\text { Cultivos anuales o } \\
\text { temporales }\end{array}$ & $\begin{array}{c}\text { Cultivos permanente y } \\
\text { semi- permanente }\end{array}$ & $\begin{array}{c}\text { Pastos } \\
\text { cultivados o } \\
\text { sembrados }\end{array}$ \\
\hline \multicolumn{1}{|c|}{ País en general } & 262546 & 1045699.88 & 510129.01 & 1352953.81 \\
\hline De $0.5 \mathrm{mz}$ a menos & 31804 & 1025.59 & 2191.96 & 29.93 \\
\hline De $0.51 \mathrm{mz}$ a $1 \mathrm{mz}$ & 16676 & 5967.06 & 4100.73 & 163.67 \\
\hline De $1 \mathrm{mz}$ a $2.50 \mathrm{mz}$ & 38215 & 39259.86 & 13873.83 & 1041.36 \\
\hline De $2.51 \mathrm{mz}$ a $5 \mathrm{mz}$ & 35672 & 68031.52 & 25732.25 & 4485.83 \\
\hline De $5.1 \mathrm{mz}$ a $10 \mathrm{mz}$ & 33686 & 99413.89 & 40597.66 & 15976.29 \\
\hline De $10.1 \mathrm{mz}$ a $20 \mathrm{mz}$ & 29881 & 122268.39 & 49647.69 & 46421.38 \\
\hline De $20.1 \mathrm{mz}$ a $50 \mathrm{mz}$ & 37440 & 214676.91 & 81516.11 & 176731.06 \\
\hline De $50.1 \mathrm{mz}$ a $100 \mathrm{mz}$ & 21238 & 167879.96 & 62810.31 & 251275.88 \\
\hline De100.1 mz a $200 \mathrm{mz}$ & 10911 & 120102.76 & 50990.37 & 276789.18 \\
\hline De $200.1 \mathrm{mz}$ a $500 \mathrm{mz}$ & 5469 & 92236.06 & 48332.52 & 316440.89 \\
\hline De $500 \mathrm{mz}$ a más & 1554 & 114837.88 & 130335.58 & 263598.34 \\
\hline
\end{tabular}

Fuente: Elaboración propia en base al IV Censo Agropecuario 2011.

Esta tabla muestra parte de las superficies utilizadas y la forma de explotación de las mismas, en manzanas de tierra. Se contabilizan desde 1/2 manzana hasta 500 y más observando que el territorio Nicaragüense usa y explota al máximo sus parcelas.

Son los pastos naturales quienes se llevan la mayor parte de la superficie territorial con 3,287755.79 mz, esto significa que aún se conservan áreas verdes contando también con 1,139, $721.42 \mathrm{mz}$ de Bosque ${ }^{3}$.

En cada departamento se contabiliza las Explotación Agraria en miles de manzanas y se observa que son las costas del Atlántico las que cuentan con la mayor parte del territorio Nacional pero Jinotega es el departamento que se destaca entre los demás con una explotación agrícola de $30,330 \mathrm{mz}$ de productos básicos, seguido por Matagalpa con 29,041 y las Regiones Autónomas. ${ }^{4}$

Granada por el contrario es el que menos aporta con 5,616 mz. Suelo agrícola, pues es un departamento que se destaca más por el turismo.

\footnotetext{
${ }^{3}$ Véase estos datos en continuación de la tabla No. 3 en el Cuadro No 10 de IV censo Cenagro 2011. Superficie en $\mathrm{mz}$ de la tierra por tipo de Aprovechamiento, según tamaño de explotaciones agropecuarias.

4 Ídem.
} 
Revista Electrónica de Investigación en Ciencias Económicas

Facultad de Ciencias Económicas, UNAN-Managua

Uso y Explotación de las tierras en Nicaragua

Tabla No.4: Aprovechamiento de la Tierra por Departamentos.

\begin{tabular}{|l|c|c|c|c|}
\hline \multirow{2}{*}{ Departamentos } & \multicolumn{4}{|c|}{} \\
\cline { 2 - 5 } & $\begin{array}{c}\text { Total de la } \\
\text { Explotación } \\
\text { Agropecuaria }\end{array}$ & $\begin{array}{c}\text { Cultivos anuales o } \\
\text { temporales }\end{array}$ & $\begin{array}{c}\text { Cultivos } \\
\text { permanente y } \\
\text { semi- permanente }\end{array}$ & $\begin{array}{c}\text { Pastos cultivados o } \\
\text { sembrados }\end{array}$ \\
\hline Nueva Segovia & 17739 & 56185.17 & 31310.71 & 26224.16 \\
\hline Jinotega & 30330 & 116566.87 & 88303.17 & 57785.03 \\
\hline Madriz & 13744 & 28445.69 & 17634.22 & 17426.90 \\
\hline Estelí & 10951 & 39253.18 & 6583.23 & 32190.49 \\
\hline Chinandega & 15368 & 73071.23 & 75518.75 & 65959.62 \\
\hline León & 18274 & 102574.13 & 9760.08 & 86301.97 \\
\hline Matagalpa & 29041 & 117979.54 & 67724.25 & 112072.26 \\
\hline Boaco & 12487 & 50711.72 & 12901.84 & 84744.00 \\
\hline Managua & 13131 & 48747.22 & 19328.52 & 50152.28 \\
\hline Masaya & 14905 & 22449.82 & 16487.00 & 4357.67 \\
\hline Chontales & 8366 & 20323.75 & 5436.02 & 142073.55 \\
\hline Granada & 5616 & 27246.20 & 13031.43 & 10020.94 \\
\hline Carazo & 7959 & 17045.06 & 12570.79 & 14500.02 \\
\hline Rivas & 12242 & 29534.10 & 25049.35 & 31335.77 \\
\hline Rio San Juan & 9138 & 49008.73 & 20818.11 & 107718.04 \\
\hline RAAN & 20541 & 124721.66 & 38322.53 & 175872.92 \\
\hline RAAS & 22714 & 121835.81 & 49349.01 & 334218.19 \\
\hline
\end{tabular}

Fuente: Elaboración propia en base al IV Censo Agropecuario 2011.

Se puede observar en la tabla No.4 las cantidades exactas que posee cada departamento en $\mathrm{mz}$ de tierra, las cuales tienen distinto forma de uso y explotación, por tanto es indispensable registrarlas año con año para reconocer el estado en el que se encuentran ${ }^{5}$.

Durante el año agrícola 2010 - 2011 se sembraron uno o más cultivos de granos básicos aprovechando los ciclos agrícolas para la obtención de los mismos. Entre ellos se destacan: El Maíz, Frijol, Arroz de riego, Arroz de secano, Sorgo Rojo, Sorgo millón y Sorgo blanco ${ }^{6}$.

El total de las explotaciones en estos rubros es de 262,546 mz de producción P/E: De los productores que tienen entre 0.5 y $1 \mathrm{mz}$ de tierra se logró la cosecha variada

\footnotetext{
${ }^{5}$ Véase estos datos en continuación de la tabla No. 3 en el Cuadro No 10 de IV censo Cenagro 2011. Superficie en $\mathrm{mz}$ de la tierra por tipo de Aprovechamiento, según tamaño de explotaciones agropecuarias.

${ }^{6}$ Véase el cuadro No. 14 del IV Censo Agropecuario 2011. Número de explotaciones agropecuarias que sembraron uno o más de granos básicos durante el año agrícola 2010-2011, según tamaño de las EA.
} 
Revista Electrónica de Investigación en Ciencias Económicas

Facultad de Ciencias Económicas, UNAN-Managua

Uso y Explotación de las tierras en Nicaragua

de 2,376 mz de los granos básicos antes mencionados; se observa que son los productores que tienen entre 20 y 50 manzanas de tierra los que siembran y cosechan más con un total de 37,440 mz de Explotación agropecuaria y 30,992 mz de uno o más cultivos ${ }^{7}$.

El maíz fue el grano que más se cosecho en este periodo con 166,567 mz de producción, seguido por el frijol con $137,879 \mathrm{mz}$ y el arroz de secano con 23,578 mz. Sin embargo hay departamentos que no cuentan con esta producción ya sea debido al clima o porque son lugares que simplemente no son aptos para este tipo de rubros, tal caso es el de Nueva Segovia, Jinotega, Madriz, Estelí, donde probablemente no se da la cosecha de Arroz debido a su clima fresco y altura a nivel del mar.

Aun así este el Arroz de riego, arroz de secano y los tipos de sorgo se cosechan en pocas cantidades en cualquiera de los departamentos, pues cada uno se centra en su potencial económico, al parecer estos no son de indispensable comercio para los que lo producen. Por lo tanto se recurre a importar debido a la falta de granos para el abastecimiento de la población.

O simplemente no es beneficioso para las y los productores, probablemente por el proceso que conlleve este, por la falta de agua como es el caso de arroz de riego o por el sin número de inconvenientes que se presentan.

Anteriormente se hacía énfasis en la Explotación Agropecuaria con 10 más cultivos de granos básicos, ahora se hará según el tamaño de las explotaciones agropecuarias, basándose en las superficies en Mz sembradas.

El total de la superficie sembrada con granos básicos es de 911,177.35 mz, destacándose siempre los productores que tienen entre 20 y $50 \mathrm{mz}$ de territorio. ${ }^{8}$ Destacando siempre la cosecha de Maíz con 100,864.67 mz.

Otros cultivos sembrados durante este mismo periodo son: La yuca, el quequisque, malanga, tomate, pipián, sandia, ayote, tabaco, chiltoma, papa, cebolla, repollo y otros $^{9}$.

\footnotetext{
${ }^{7}$ En cada EA se puede haber sembrado uno o más cultivos de granos básicos en el ciclo agrícola.

${ }^{8}$ Véase Cuadro No. 15 del IV Censo agropecuario 2015. Superficie en mz sembradas con granos básicos durante el año agrícola 2010-2011, Según el tamaño de las Explotaciones agropecuarias.

9 Ídem, cuadro No. 19. Superficie en $\mathrm{mz}$ sembradas de otros cultivos.
} 
Revista Electrónica de Investigación en Ciencias Económicas

Facultad de Ciencias Económicas, UNAN-Managua

Uso y Explotación de las tierras en Nicaragua

La yuca es el abarrote más producido y comercializado al contabilizar 31,389.75 de áreas cultivadas. Este tipo de cultivos es considerando semi- perenne por su forma de conservarse ante los cambios del medio ambiente.

Todos son productos de Comercio puesto que son nutritivos y son cosechados durante todo el año.

La superficie en $\mathrm{mz}$ sembradas con cultivos permanentes y semi- permanentes es de 418,870.66 mz aproximadamente. Estos cultivos son: El café, las Musáceas, la caña de azúcar, el cacao, cítricos, coco, mango, piña, aguacate, palma africana, pitahaya y otros.

Todos ellos se obtienen en épocas del año no en todo el año; y en este periodo se cosecho en superficie mayoritariamente como es siempre el café, pues es el rubro de mayor comercio internacional en su temporada la cual se da una vez por año y su comercialización es buena siempre y cuando no hayan externalidades negativas.

$180,219.72 \mathrm{mz}$ es la superficie utilizada a nivel nacional, donde se produce este rubro, principalmente en la región central y norte de Nicaragua.

Es necesario señalar que la tierra alberga recursos estratégicos vitales para el desarrollo presente y futuro de Nicaragua, por lo tanto, su gestión, administración y uso requieren de políticas de tierras con una visión integral que promuevan y equilibren los criterios sociales, ambientales, económico-productivos y culturales ${ }^{10}$.

Según la aplicación de la Reforma Agraria, en su art.2 se declaran afectas a) Las propiedades ociosas 0 deficientemente explotadas en manos de personas naturales o jurídicas que sean propietarios de más de 500 manzanas, en la Zona A, y más de 1,000 manzanas en la Zona B.

b) Las tierras que a la fecha de emisión de la presente Ley estén dadas en arriendo o cedidas bajo cualquier otra modalidad, cuyos propietarios posean más de 500 manzanas en la Zona A, y más de 1,000 manzanas en la Zona B.

\footnotetext{
${ }^{10}$ Marco General de Políticas de Tierras; Decreto No. 70-2006, Aprobado el 30 de Octubre del 2006. Publicado en La Gaceta No. 217 del 08 de Noviembre del 2006
} 
Revista Electrónica de Investigación en Ciencias Económicas

Facultad de Ciencias Económicas, UNAN-Managua

Uso y Explotación de las tierras en Nicaragua

c) Las propiedades en abandono.

En su art.3 manifiesta que el límite de 500 o 1000 manzanas se calculará sumando el área de las propiedades que posea una misma persona natural o jurídica, aunque estas se encuentren geográficamente en distintos lugares ${ }^{11}$.

\section{Conclusión}

El tema de Uso y explotación de tierras en Nicaragua juega un papel sumamente importante dentro de la economía de Nicaragua, por tanto es necesario hacer pautas que fijen políticas que apunten al desarrollo integral, económico y agrícola de la sociedad. Esto implica la necesidad un marco de posibilidades para mejorar la calidad de vida de hombres y mujeres en igualdad de condiciones.

Lo expuesto invita a pensar en la situación en que se encuentra la tierra en nuestro país y esto hace referencia a hacer conciencia de cómo deberíamos hacer uso de ella, tomando en cuenta su potencial y no explotándola indebidamente.

\footnotetext{
${ }^{11}$ Ley de Reforma Agraria; Decreto No. 782. fundamentada en el Arto. 23 del Decreto No 388 del 2 de Mayo de 1981; República de Nicaragua.
} 


\section{Revista Electrónica de Investigación en Ciencias Económicas \\ Facultad de Ciencias Económicas, UNAN-Managua \\ Uso y Explotación de las tierras en Nicaragua}

\section{Bibliografía}

INIDE-MAGFOR. (2011). IV Censo Nacional Agropecuario CENAGRO.

Vivas, Viachica, E. (2005). Análisis de la utilización del recurso suelo en Nicaragua. Revista La CALERA; UNA; Pág. 1-6.

Rodan, H. (2002). Estado actual de la Información sobre Recursos Forestales y cambio en el uso de la tierra. Revista "Estado de la información forestal en Nicaragua”. Pág.177.

INETER. (2008). Caracterización Geográfica del Territorio Nacional, recuperado el 25 de mayo del 2015, de http://www.ineter.gob.ni/caracterizacióngeografica/capitulo6.html

MAGFOR- INIDE. (2013) Uso potencial de la tierra comprendido en mapas IV Censo Agropecuario (CENAGRO). Managua-Nicaragua.

MAGFOR. (2001), Mapa forestal de Nicaragua, 2000, conjunto de mapas, ManaguaNicaragua

MARENA. (2006). Informe Nacional de áreas protegidas de Nicaragua, ManaguaNicaragua

Marco general de políticas de tierra (2006). "Ley de reforma agraria" capítulo I Managua-Nicaragua. 